\title{
Morphine and diprenorphine together potentiate responding for positively reinforcing brain stimulation
}

\author{
JAMES M. LAGASSE, LOUIS M. WETSTEIN, STEPHANIE A. CZIRR, and LARRY D. REID \\ Rensselaer Polytechnic Institute, Troy, New York
}

\begin{abstract}
Rats fixed with chronically indwelling bipolar electrodes for stimulation of the lateral hypothalamus were trained to press for brain stimulation. Subsequent to training, they were allowed to press for $30 \mathrm{~min}$ a day for a moderate intensity of stimulation until response rates stabilized. Then, prior to some opportunities to press, the rats were given either a $10-\mathrm{mg} / \mathrm{kg}$ dose of morphine sulfate or a 30- $\mu \mathrm{g} / \mathrm{kg}$ dose of diprenorphine (an antagonist of morphine's analgesia and behavioral suppression), or both. The large dose of morphine suppressed responding, and the small dose of diprenorphine facilitated responding, replicating previous observations. Morphine plus diprenorphine produced marked increments in pressing. These data provide further support for the idea that morphine's capacity to facilitate responding for brain stimulation is unrelated to its analgesic properties. Also, the data support the idea of a separate opioid receptor for opioid reward.
\end{abstract}

The study of the effects of addicting drugs on pressing for intracranial stimulation (ICS) has been instrumental in the development of modern theories of addictions (for a germinal review, see Esposito \& Kornetsky, 1978). One of the central ideas among those forming the newer concept of addictions is the idea that addicting drugs have the unique capability of establishing neural activity in the same tissue for which direct electrical stimulation is positively reinforcing (Wise, 1983). Despite the saliency of this idea, the data on which it is based do not coalesce into simple structures. The effects of morphine (MOR) on pressing for ICS, for example, are characterized by a complex interaction of dose, time after dosing, and days of daily dosing.

When large doses of MOR (e.g., $10 \mathrm{mg} / \mathrm{kg}$ ) are given to rats pressing for ICS, the effect seen shortly after injections is suppression of pressing (Olds \& Travis, 1960). If, however, measures are made some time after large doses (e.g., $4.0 \mathrm{~h}$ ), pressing is increased (Adams, Lorens, \& Mitchell, 1972). With continuance of large daily doses, the initial suppressive effects wane and the period of facilitation moves closer and closer to the time of injection (Bush, Bush, Miller, \& Reid, 1976). Small doses of MOR (e.g., $1.0 \mathrm{mg} / \mathrm{kg}$ ) and other addicting opioids can produce facilitated pressing without a period of suppressed pressing (Gerber, Bozarth, \& Wise, 1981; Reid, in press).

There are a number of issues associated with the doseresponse relationship between MOR and pressing for ICS. One issue is whether or not the initial depression seen with

\footnotetext{
Thanks are extended to Bruce McCutcheon, from the Department of Psychology, SUNY at Albany, for reviewing an earlier version of this paper, and for generally being helpful throughout the project. The completion of the project was aided by Grant AA06212 from the NIAAA. The authors' mailing address is Department of Psychology, Rensselaer Polytechnic Institute, Troy, NY 12180.
}

large doses is due to MOR's potential to fully activate the reward system thus making ICS superfluous (e.g., the relevant receptors may be saturated). Another possibility is that the large-dose suppression is merely a product of incapacitating side effects such as drug-induced catatonia. Since the potentially incapacitating side effects show tolerance with repeated dosing, such tolerance could account for the shifts in patterns of pressing with repeated dosing. Such an explanation is compatible with the view that MOR's potential for inducing signs of positive affect (euphoria?) does not tolerate with repeated dosing (Bush et al., 1976; Esposito \& Kornetsky, 1977; Kelley \& Reid, 1977). There is also the possibility that MORfacilitated pressing seen after a passage of time after large doses is a result of an essential rebound or opponent process (but see Bermudez-Rattoni, Cruz-Morales, \& Reid, 1983; Collaer, Magnuson, \& Reid, 1977; Gerber et al., 1981).

Diprenorphine (DIP) is an agent with high affinity for opioid receptors (opioceptors) (Chang, Hazum, \& Cuatrecasas, 1980, 1981). DIP antagonizes opioid-induced analgesia and catatonia. In many in vitro bioassays, DIP acts as an antagonist. Yet, in the intact rat, DIP leads to increased pressing for ICS and is capable of establishing a conditioned place preference (Beaman, Hunter, \& Reid, 1984; Pollerberg, Costa, Shearman, Herz, \& Reid, 1983). Although DIP and naloxone (the prototypic antagonist at opioceptors) share the ability to block opioid analgesia, they produce different effects with respect to pressing for ICS. Naloxone blocks the capability of small doses of MOR to enhance pressing (Bozarth \& Reid, 1977). Also, naloxone produces a reduction in (but does not eliminate) pressing for ICS in rats with no other opioids, presumably due to naloxone's ability to antagonize the effects of endogenous opioids (Stapleton, Merriman, Coogle, 
Gelbard, \& Reid, 1979). Furthermore, naloxone, being the broad spectrum antagonist, blocks DIP's capacity to enhance pressing (Pollerberg et al., 1983).

DIP's capacity to antagonize many of MOR's effects while at the same time having a potential for not antagonizing MOR's capacity to potentiate pressing for ICS (since it produces not the opposite, but the same general effects as MOR) represents an interesting opportunity to test an issue associated with MOR's effects on pressing for ICS. If DIP and a large dose of MOR together enhance pressing for ICS, then it is difficult to hold that the large dose of MOR is suppressing response rates because maximum rewarding effects have been achieved. In other words, if pressing is increased under the combined effects of a large dose of MOR and a dose of DIP, then the system's capability is not saturated, but has further capacity.

\section{METHOD}

\section{Subjects}

Six male Sprague-Dawley rats were purchased from Taconic Farms (Germantown, NY) when they weighed about $300 \mathrm{~g}$. They were housed in individual cages with food and water always available in a windowless room that was maintained at $24^{\circ} \mathrm{C}$ and had $12 \mathrm{~h}$ of artificial light/day.

Each rat was fixed, using standard stereotaxic procedures that included deep anesthesia (Nembutal, $50 \mathrm{mg} / \mathrm{kg}$ ), with a chronically indwelling bipolar electrode (Plastic Products, Ms 303). The electrode's stimulating tip was aimed for the medial forebrain bundle as it courses through the lateral hypothalamus. The stereotaxic coordinates were $3.2 \mathrm{~mm}$ posterior to bregma, $1.6 \mathrm{~mm}$ lateral to the midline, and $8.5 \mathrm{~mm}$ below the surface of the skull, with the electrode's shaft perpendicular to a horizontal plane between bregma and lambda.

Following behavioral testing and deep anesthesia, the rats were perfused intracardially with saline followed by $10 \%$ formalin. Brains were removed and soaked in a formalin-sucrose solution for 1 week, frozen, blocked, and sliced in $40-\mu$ sections. Wet sections were placed on slides covered with glycerin and a coverslip. Sites were further identified by using the slides and a projection microscope to trace images of brain structures and location of electrodes. All six electrode tips were clustered in a small area lateral to the plane of descending fornix, medial to the internal capsule, and just below the zona incerta at the anterior-posterior plane of the ventro medial $\mathrm{n}$, that is, the medial forebrain bundle of the lateral hypothalamus.

In summary, 6 rats living in standard laboratory conditions and fixed with electrodes for ICS of the medial forebrain bundle served as subjects.

\section{Apparatus}

All testing was in $24 \times 30 \times 38 \mathrm{~cm}$ chambers in which a $2.5 \times 2 \mathrm{~cm}$ lever extended through one wall. The leads from the ICS generator to the rat involved a slip-ring assembly, thereby allowing the rat to move freely in the chamber with the possibility of ICS. Each depression of the lever yielded a single ICS (fixed ratio 1:1) of 60Hz sine waves of $0.3 \mathrm{sec}$ of varying intensities but always less than $50 \mu \mathrm{A}$ (RMS). If, however, a rat pressed during an ICS, that press was recorded but no ICS was delivered.

\section{Procedure}

The rats were fixed with electrodes, allowed 5 days to recover from surgery, and then trained to press for ICS. During initial daily sessions of pressing, intensity of ICS was varied to allow for the selection of an intensity that would maintain a relatively steady rate of pressing of about 2,000 presses in a 30-min session. This rate allows for both marked increases and marked decreases in pressing.

After the selection of intensities, rats were tested once a day during the middle $4 \mathrm{~h}$ of the light cycle. After 10 self-administered ICSs, the number of self-delivered ICSs during $30 \mathrm{~min}$ was tabulated automatically. The rats were tested daily under this regimen, with only minor changes in intensity of ICS, until press rates stabilized (dayto-day fluctuations, across 3 days, no more than 10\%); stabilization occurred for all rats within 14 days. The final intensities ranged from 14 to $34 \mu \mathrm{A}$ (RMS) which generated a mean of 2,054 presses/30 min on the $1 \mathrm{st}$ day of the procedures associated with testing for drug effects. Once an intensity of ICS was set for a rat during these preliminaries, that intensity remained the same throughout the balance of the procedures.

With stabilization of pressing rates and with continuance of the daily regimen, a series of injections were given. There were four kinds of injections, placebo (two injections of the carrier of drugs, saline) and three kinds involving opioids: (1) a $10-\mathrm{mg} / \mathrm{kg}$ injection of MOR sulfate and an injection of saline; (2) a $30-\mu \mathrm{g} / \mathrm{kg}$ injection of DIP hydrochloride and an injection of saline; and (3) an injection of both MOR and DIP at the same doses as those given with the saline injections. The injections, in volumes of $1 \mathrm{ml} / \mathrm{kg}$, were given subcutaneously, one immediately after the other, $15 \mathrm{~min}$ before testing. The choices of doses came from previous dose-response studies. The dose of DIP was a dose clearly antagonizing all signs of opioid analgesia (Pollerberg et al., 1983). The dose of MOR was a commonly used dose and the dose of many initial studies with MOR and pressing for ICS (e.g., Adams et al., 1972; Bush et al., 1976).

Each kind of opioid administration was given to each rat once before one of the daily tests. Each rat's full testing spanned 8 consecutive days, but each rat began on a different day (just after achieving stable pressing), thereby assuring that, across rats, opioids were given on different days, thus controlling for any potential effects associated with a given day. The order of kinds of injections, across consecutive days, followed the pattern of POP POP PO, where P stands for placebo and $O$ stands for one of the three kinds of opioid administrations. Two rats received each kind of opioid first and order of further administrations was arranged as a Latin square. Since, upon initial inspection of the results, we could discern no drug order effect, order was ignored in subsequent analyses.

An analysis of variance for repeated measures across scores of predrug placebos yielded an $F(2,10)=0.25, p>.50$, indicating no systematic effects associated with days of testing when no opioids were given. Furthermore, postdrug placebo scores were similar to the predrug scores. In other words, pressing remained stable unless under the influence of opioids. To assess the effects of the opioids, the scores of kind of opioid administration were compared with the scores obtained with the placebo administered immediately preceding the opioid administration. Statements of statistical significance of these comparisons (Figure 1) are based on the $t$ tests for dependent (repeated) measures.

\section{RESULTS AND DISCUSSION}

With reference to predrug-placebo performance, all 6 subjects showed increased pressing under the influence of DIP, decreased pressing under MOR, and increased pressing under DIP plus MOR (Figure 1). Also, across all 6 subjects, the scores produced by DIP plus MOR were higher than the scores produced by DIP by itself (Figure 1). The comparison of difference scores for DIP alone with those of DIP plus MOR yielded a $t(5)=5.27$, $p<.01$. 


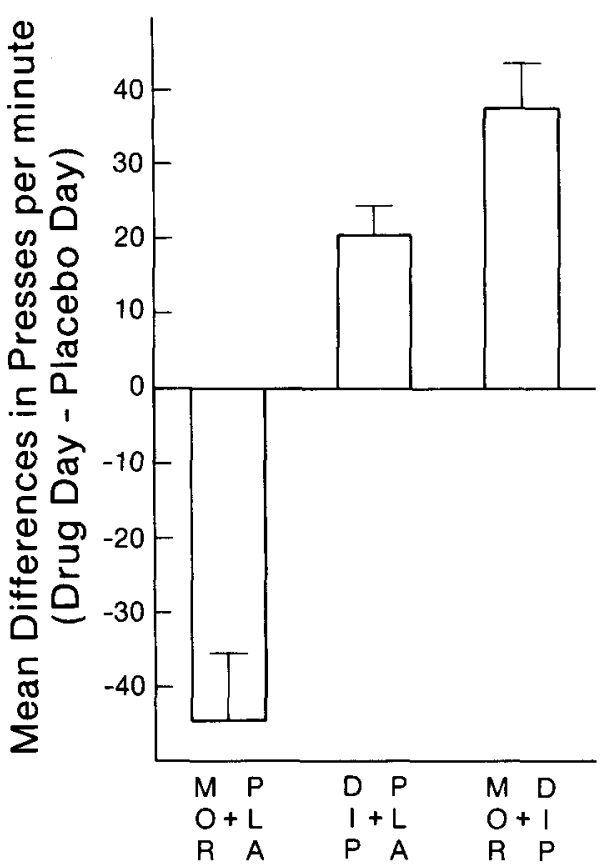

Figure 1. The mean differences from predrug placebo are depicted with standard errors of the mean. The large dose of MOR plus placebo reliably reduced pressing $[t(5)=5.08, p<.01]$, the dose of DIP increased pressing $[t(5)=5.90, p<.01]$, and the two opioids together increased pressing $[t(5)=6.29, p<.01]$.

A large dose of MOR combined with an effective dose of DIP produced more pressing than placebos or either drug alone. This finding indicates that opioids' potentiation of pressing for ICS is not a product of opioids' capacity to elicit analgesia, behavioral depression, or many of the effects of MOR antagonized by DIP. DIP at this dose clearly antagonizes opioid analgesia (Pollerberg et al., 1983). The potentiated pressing is not a product of rebound from some initial suppression, since the combination of MOR and DIP did not produce initial suppression.

The large-MOR-dose effect on pressing for ICS does not appear to be due to saturation of relevant receptors, because, if all binding sites were occupied with the large dose of MOR, then the addition of more opioid molecules (e.g., DIP) would not have a site available for further action. Yet we clearly see an increment in pressing with the addition of DIP. The increment seen with DIP is best accounted for by presuming that DIP's antagonist features block the debilitating effects of large doses of MOR, thereby "releasing" the rat to press unencumbered.

These data support the idea (Bermudez-Rattoni et al., 1983; Pollerberg et al., 1983) that there is a separate type of receptor, of the general class of opioceptors (receptors sensitive to naloxone), related to indices of opioid reward. That receptor mediates events unrelated to opioid analgesia, opioid catatonia, and opioid behavioral suppression. The present data complement those of Bermudez-
Rattoni et al., showing that the combination of MOR plus another antagonist of analgesia (WIN 44,441) leads to pressing rates greater than the larger dose of MOR by itself. These data, however, also show that MOR and an antagonist (DIP) together produce more pressing than either alone. Both unpublished data and the data of Pollerberg et al. also indicate that fentanyl (a short-acting opioid analgesic) and DIP together produce greater enhancement of pressing than does either one alone. This facilitating effect of DIP is in marked contrast to the effects of naloxone, which blocks all agonist effects, including those of DIP (Pollerberg et al., 1983).

The following observations are supportive of the general idea (Bush et al., 1976; Farber \& Reid, 1976; Rossi \& Reid, 1976) that opioid analgesia and opioid reward are separable. (1) MOR, heroin, fentanyl, and other addicting opioids enhance responsiveness to rewarding brain stimulation. (2) Some opioids that are clearly antagonists with respect to opioid analgesia also enhance pressing for a fixed intensity of ICS (Bermudez-Rattoni et al., 1983; Pollerberg et al., 1983; this paper). (3) Some opioids, for example, DIP, that are antagonists to analgesia show other signs of eliciting positive affect (Beaman et al., 1984). (4) Some opioid analgesics, for example, the negative optical isomer of ethylketocyczine, show no sign of enhancing pressing for ICS (Reid, Hubbell, Dunn, Hunter, \& Costa, 1985). (5) MOR plus a dose of DIP, which clearly blocks opioid analgesia, enhances pressing more than DIP alone (this paper). And (6) most of these effects have been shown to be sensitive to naloxone's antagonism. The idea of separability of opioid reward and opioid analgesia also finds strong support in the anatomical studies of Bozarth (1983). From studies using rats, it is concluded that opioids' potential for addiction is related to the addicting opioids' ability to induce signs of an enhanced positive affect (a condition for MOR's positively reinforcing effects) that is mediated by way of a distinct type of opioceptor (distinct from those of analgesia and catatonia) located in the ventral tegmental area and involves systems of the medial forebrain bundle.

\section{REFERENCES}

Adams, W. J., LoRens, S. A., \& Mitchell, C. L. (1972). Morphine enhances lateral hypothalamic self-stimulation in the rat. Proceedings of the Society for Experimental Biology and Medicine, 140, 770-771.

Beaman, C. M., Hunter, G. A., \& ReID, L. D. (1984). Diprenorphine, an antagonist of opioid analgesia, elicits a positive affective state in rats. Bulletin of the Psychonomic Society, 22, 354-355.

Bermudez-Rattoni, F., Cruz-Morales, S., \& Reid, L. D. (1983). Addictive agents and intracranial stimulation (ICS): Novel antagonist and agonists of morphine and pressing for ICS. Pharmacology Biochemistry \& Behavior, 18, 777-784.

BozARTH, M. A. (1983). Opiate reward mechanisms mapped by intracranial self-administration. In J. E. Smith \& J. D. Lane (Eds.), Neurobiology of opiate reward mechanisms (pp. 331-359). Amsterdam: Elsevier Biomed Press/North Holland.

Bozarth, M. A., \& ReID, L. D. (1977). Addictive agents and intracranial stimulation (ICS): Naloxone blocks morphine's acceleration of pressing for ICS. Bulletin of the Psychonomic Society, 10, 478-480. 
Bush, E. D., Bush, M. F., Miller, M. A., \& Reid, L. D. (1976). Addictive agents and intracranial stimulation: Daily morphine and lateral hypothalamic self-stimulation. Physiological Psychology, 4, 79-85.

Chang, K. J., Hazum, E., \& Cuatrecasas, P. (1980). Possible role of distinct morphine and enkephalin receptors in mediating actions of benzomorphan drugs (putative kappa and sigma agonists). Proceedings of the National Academy of Science, USA, 77, 4469-4473.

Chang, K. J., Hazum, E., Cuatrecasas, P. (1981). Novel opiate binding sites selective for benzomorphan drugs. Proceedings of the National Academy of Science, USA, 78, 4141-4145.

Collaer, M. L., Magnuson, D. J., \& ReID, L. D. (1977). Addictive agents and intracranial stimulation (ICS): Pressing for ICS before and after self-administration of sweetened morphine solutions. Physiological Psychology, 5, 425-428.

Esposito, R., \& KoRNETSKY, C. (1977). Morphine lowering of selfstimulation thresholds: Lack of tolerance with long term administration. Science, 195, 189-191.

Espostro, R., KoRnetsky, C. (1978). Opioids and rewarding brain stimulation. Neuroscience \& Biobehavioral Reviews, 2, 115-122.

FARBER, P. D., \& REID, L. D. (1976). Addictive agents and intracranial stimulation (ICS): Daily morphine and pressing for combinations of positive and negative ICS. Physiological Psychology, 4, 262-268.

Gerber, G. J., Bozarth, M. A., \& WiSe, R. A. (1981). Small-dose intravenous heroin facilitates hypothalamic self-stimulation without response suppression in rats. Life Sciences, 28, 557-562.

KeLLEY, K. L., \& REID, L. D. (1976). Addictive agents and intracranial stimulation: Morphine and thresholds for positive intracranial reinforcement. Bulletin of the Psychonomic Society, 10, 298-300.
Olds, J., Travis, R. P. (1960). Effects of chlorpromazine, meprobamate, pentobarbital and morphine on self-stimulation. Journal of Pharmacology \& Experimental Therapeutics, 128, 397-404.

Pollerberg, G. E., Costa, T., Shearman, G. T., Herz, A., \& Reid, L. D. (1983). Opioid antinociception and positive reinforcement are mediated by different types of opioid receptors. Life Sciences, 33, 1549-1559.

REID, L. D. (in press). Tests involving pressing for intracranial stimulation as an early procedure for screening likelihood of addiction of opioids and other drugs. In M. A. Bozarth (Ed.), Methods of assessing the reinforcing properties of abused drugs. New York: SpringerVerlag.

Reid, L. D., Hubbell, C. L., DunN, L. L., Hunter, G. A., \& CostA, T. (1985). Selected opioids and responding for intracranial reinforcement. Neuropeptides, 5, 331-334.

Rossi, N. A., \& REID, L. D. (1976). Affective states associated with morphine injections. Physiological Psychology, 4, 269-274.

Stapleton, J. M., Merriman, V. J., Coogle, C. L., Gelbard, S. D., \& REID, L. D. (1979). Naloxone reduces pressing for intracranial stimulation of sites in the periaqueductal gray area, accumbens nucleus, substantia nigra, and lateral hypothalamus. Physiological Psychology, 7, 427-436.

Wise, R. A. (1983). Brain neuronal systems mediating reward processes. In J. E. Smith \& J. D. Lane (Eds.), Neurobiology of opiate reward mechanisms (pp. 405-437). Amsterdam: Elsevier Biomedical Press.

(Manuscript received February 3, 1987; revision accepted for publication May 23, 1987.) 